\title{
Le contexte de l'initiation sexuelle en évolution dans la région subsaharienne de l'Afrique
}

Barbara Mensch

Population Council

Follow this and additional works at: https://knowledgecommons.popcouncil.org/departments_sbsr-pgy

Part of the Demography, Population, and Ecology Commons, Family, Life Course, and Society Commons, Gender and Sexuality Commons, International Public Health Commons, Medicine and Health Commons, and the Sociology of Culture Commons

How does access to this work benefit you? Let us know!

\section{Recommended Citation}

Mensch, Barbara. 2007. "Le contexte de l'initiation sexuelle en évolution dans la région subsaharienne de l'Afrique," Promouvoir la santé, la sécurité et la productivité transitions vers l'âge adulte Document no. 26. New York: Population Council. 


\section{Le contexte de l'initiation sexuelle en évolution dans la région subsaharienne de l'Afrique}

\section{Préparé par Barbara S. Mensch}

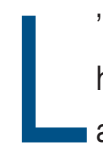

'ampleur de l'épidémie du SIDA en Afrique subsaharienne expose particulièrement les jeunes femmes au risque de l'infection au VIH et requiert une meilleure compréhension des comportements reproductifs, y compris l'initiation sexuelle et les relations sexuelles pré-maritales. Au cours des 20 dernières années, on note, en Afrique subsaharienne, une importante réduction de la proportion des jeunes femmes qui se marient pendant l'adolescence.

Un récent rapport des politiques (Cohen 2004) sur les habitudes reproductives des adolescents affirme que la croissance des relations sexuelles pré-maritales est une conséquence inévitable des mariages tardifs dans les pays en développement, en particulier à cause du fait que la période pendant laquelle les filles sont sexuellement mûres et non mariées est prolongée quand le mariage est retardé (Alan Guttmacher Institute 1998; Bongaarts et Cohen 1998). La proportion des jeunes femmes ayant eu des relations sexuelles pré-maritales a-t-elle aussi augmenté là où le mariage des adolescentes a diminué ? Ou bien la baisse des jeunes femmes qui se marient tôt a-t-elle peu d'influence sur le comportement sexuel pré-marital ? Même si la proportion des jeunes femmes ayant eu des relations sexuelles pré-maritales augmente, est-ce que l'initiation sexuelle à un âge avancé, en moyenne, est-elle une conséquence du retardement du mariage dans la jeune génération actuelle?
Compte tenu des différences possibles entre la nature de l'activité sexuelle au sein du mariage et avant celui-ci (Clark 2004), les planificateurs de programmes devraient aussi considérer qu'un changement dans le contexte de l'initiation sexuelle peut influencer le risque de l'infection du VIH parmi les jeunes femmes. Une conséquence potentielle de l'augmentation des relations sexuelles pré-maritales est la croissance de la fertilité non-désirée. Un taux élevé des filles qui ont des rapports sexuels avant le mariage peut aussi mener à un taux élevé d'avortement provoqué, démarche souvent illégale et à risque dans cette région. En outre, si l'activité sexuelle est plus fréquente parmi les adolescentes mariées que les non-mariées, si celles qui sont mariées ont beaucoup plus de relations sexuelles non protégées, les filles non-mariées ont plus souvent des partenaires multiples et plusieurs de ces partenaires ont la chance de se croiser. Ces deux circonstances sont ainsi des facteurs à risque pour les infections sexuellement transmissibles, y compris le VIH.

\section{Examiner les relations entre l'âge du mariage} et le relations sexuelles pré-maritales

Un récent projet du Population Council a fait des recherches sur les liens entre le changement de l'âge au mariage et le comportement sexuel pré-marital dans 27 pays de l'Afrique subsaharienne. Les analyses ont été faites à partir des données collectées entre 1994 et 2004 pendant des 
Tableau 1 Pourcentage des femmes enquêtées entre 20 à 24 ans et 40 à 44 ans qui ont rapporté être mariées et avoir eu des relations sexuelles avant l'âge de 18 ans, 27 pays de la région subsaharienne de l'Afrique

\begin{tabular}{|c|c|c|c|c|c|c|c|c|}
\hline \multirow[b]{2}{*}{ Pays et année du sondage } & \multicolumn{2}{|c|}{$\begin{array}{l}\text { Mariées avant } \\
18 \text { ans }^{\mathrm{a}}\end{array}$} & \multicolumn{2}{|c|}{$\begin{array}{l}\text { Relations sexuelles } \\
\text { avant } 18 \text { ans }^{\mathrm{a}}\end{array}$} & \multicolumn{2}{|c|}{$\begin{array}{l}\text { Relations sexuelles } \\
\text { pré-maritale } \\
\text { avant } 18 \text { ans }^{\text {b }}\end{array}$} & \multicolumn{2}{|c|}{$\begin{array}{l}\text { Mariées avant } \\
18 \text { ans sans } \\
\text { relations sex- } \\
\text { uelles antériors }\end{array}$} \\
\hline & $20-24$ & $40-44$ & $20-24$ & $40-44$ & $20-24$ & $40-44$ & $20-24$ & $40-44$ \\
\hline \multicolumn{9}{|l|}{ Afrique de l'Ouest / centrale } \\
\hline Bénin 2001 & 36,7 & 43,6 & 63,5 & 61,0 & 38,8 & 24,0 & 24,7 & 37,0 \\
\hline Burkina Faso 2003 & 51,9 & 59,0 & 65,3 & 68,2 & 22,7 & 12,7 & 42,5 & 55,5 \\
\hline Cameroun 2004 & 47,2 & 57,2 & 70,3 & 76,5 & 35,1 & 28,8 & 35,2 & 47,7 \\
\hline République Centrafricaine 1994-95 & 57,0 & 64,6 & 81,0 & 81,5 & 42,3 & 30,4 & 38,7 & 51,2 \\
\hline Tchad 1996-97 & 71,4 & 77,9 & 77,3 & 81,4 & 14,8 & 10,5 & 62,5 & 70,9 \\
\hline Gabon 2000 & 33,6 & 41,0 & 81,0 & 83,9 & 62,3 & 60,2 & 18,7 & 23,7 \\
\hline Ghana 2003 & 27,9 & 37,7 & 46,4 & 56,3 & 24,3 & 27,1 & 22,0 & 29,2 \\
\hline Guinée 1999 & 64,5 & 68,5 & 77,6 & 78,5 & 24,4 & 15,5 & 53,2 & 63,0 \\
\hline Côte d'Ivoire 1998 & 33,2 & 49,7 & 75,8 & 78,1 & 54,7 & 41,3 & 21,1 & 36,8 \\
\hline Mali 2001 & 65,4 & 66,0 & 79,3 & 77,2 & 26,0 & 17,6 & 53,4 & 59,6 \\
\hline Niger 1998 & 76,6 & 89,1 & 79,1 & 89,8 & 4,5 & 2,7 & 74,5 & 87,1 \\
\hline Nigeria 2003 & 43,3 & 65,1 & 57,0 & 74,0 & 19,0 & 12,5 & 38,0 & 61,5 \\
\hline Sénégal 1997 & 36,1 & 61,9 & 43,7 & 65,8 & 9,8 & 3,9 & 33,9 & 61,8 \\
\hline Togo 1998 & 30,5 & 40,4 & 65,0 & 59,5 & 47,4 & 29,9 & 17,6 & 29,6 \\
\hline \multicolumn{9}{|l|}{ Afrique du Sud / australe } \\
\hline Comores 1996 & 29,7 & 54,5 & 34,8 & 63,6 & 9,6 & 10,8 & 25,2 & 52,8 \\
\hline Éthiopie 1999 & 49,1 & 79,4 & 51,6 & 81,4 & 7,5 & 6,7 & 44,1 & 74,6 \\
\hline Kenya 2003 & 24,6 & 37,2 & 50,3 & 65,9 & 36,9 & 43,8 & 13,4 & 22,1 \\
\hline Madagascar 1997 & 40,4 & 49,5 & 65,8 & 68,3 & 40,5 & 31,8 & 25,3 & 36,5 \\
\hline Malawi 2000 & 46,9 & 55,7 & 65,4 & 68,8 & 31,7 & 25,1 & 33,7 & 43,7 \\
\hline Mozambique 2003 & 55,9 & 55,9 & 81,2 & 73,5 & 48,1 & 34,5 & 33,1 & 39,0 \\
\hline Namibie 2000 & 9,8 & 15,8 & 49,1 & 39,8 & 42,2 & 26,5 & 6,9 & 13,4 \\
\hline Rwanda 2000 & 19,5 & 21,5 & 26,9 & 26,8 & 10,2 & 7,0 & 16,7 & 19,7 \\
\hline Afrique du Sud 1998 & 7,9 & 15,1 & 55,3 & 45,8 & 50,1 & 35,4 & 5,2 & 10,4 \\
\hline Tanzanie 2004 & 41,4 & 45,2 & 63,4 & 63,3 & 37,4 & 28,3 & 26,0 & 35,0 \\
\hline Uganda 2001 & 53,9 & 59,5 & 73,5 & 78,5 & 35,9 & 35,5 & 37,6 & 43,0 \\
\hline Zambie 2001 & 42,1 & 59,7 & 66,0 & 74,6 & 38,9 & 23,9 & 27,1 & 50,7 \\
\hline Zimbabwe 1999 & 28,7 & 39,4 & 38,9 & 52,1 & 17,2 & 21,9 & 21,7 & 30,2 \\
\hline
\end{tabular}

Note : Les chiffres en gras indiquent les valeurs significatives à $p<0,05$ à la base d'un t-test pour l'égalité des moyennes.

${ }^{a}$ Calculé à partir d'une table à sortie simple.

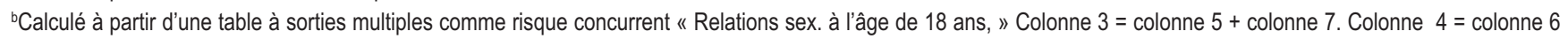
+ colonne 8.

Source : Tableaux des données des Demographic and Health Surveys.

enquêtes sur la démographie et la santé. Après avoir enregistré la réduction du mariage précoce au cours des 20 dernières années, les chercheurs se posent la question de savoir comment les réductions de la prévalence du mariage précoce affectent la possibilité de l'initiation sexuelle à l'âge de 18 ans ainsi que la possibilité d'initier les relations sexuelles pré-maritales au même âge. Ils re- cherchent aussi si les jeunes femmes transitent rapidement ou lentement vers le mariage après l'initiation sexuelle. On estime que plus l'intervalle entre l'initiation sexuelle et le mariage est long, plus le risque est grand d'avoir une grossesse non désirée, plus nombreux sont les partenaires sexuels et plus il est probable que la jeune femme soit exposée aux infections sexuelles transmissibles. Enfin, ils 
ont également étudié si l'augmentation des relations sexuelles pré-maritales est due à la plus longue période d'exposition à ces dernières entre la puberté et le mariage ou au changement des normes relatives à l'acceptation des relations sexuelles en dehors du mariage.

\section{Les résultats montrent un décalage au niveau du contexte de l'initiation sexuelle}

Bien que le mariage des enfants soit encore fréquent dans la région, les analyses indiquent que :

- La prévalence du mariage avant l'âge de 18 ans a sérieusement diminué dans 24 des 27 pays africains au sud du Sahara.

- La prévalence des relations sexuelles avant l'âge de 18 ans a fortement augmenté dans 19 pays.

- La probabilité du mariage avant l'âge de 18 ans après les relations sexuelles pré-maritales a fortement diminué dans cinq pays et reste inchangée dans les autres.

- La prévalence de l'initiation sexuelle avant l'âge de 18 ans, que ce soit avant ou après le mariage a fortement diminué dans 13 pays, augmenté dans 4 et est resté la même dans 10 pays.

En résumé, alors que la probabilité des activités sexuelles précoces est restée soit la même ou a diminué dans 23 des 27 pays, un décalage au niveau du contexte de l'initiation sexuelle du mariage à la période pré-maritale a eu lieu à travers la région. Parmi les 19 pays ayant une croissance importante en ce qui concerne la prévalence des rapports pré-maritaux, une exposition plus longue à la période de risque explique une plus grande proportion d'augmentation dans huit pays, alors qu'un taux élevé des rapports sexuels pré-maritaux explique une plus grande proportion dans cinq pays. Dans les six pays restants, la croissance est due de façon approximativement égale à un taux plus élevé de rapports sexuels et de longue exposition.

\section{Implications pour les interventions et les recherches futures}

Les planificateurs de programmes qui conçoivent les interventions visant les filles adolescentes dans les régions de l'Afrique subsaharienne doivent comprendre que bien que l'initiation sexuelle avant l'âge de 18 ans a diminué ou soit restée la même dans la majorité des pays, il y a eu un décalage dans le contexte de l'initiation sexuelle, qui, avec l'augmentation de l'âge du mariage, peut survenir avant le mariage. Ce changement peut influencer la demande d'informations et services liés à la santé reproductive parmi les filles adolescentes non mariées ; cependant la plupart des services de planification familiale et de santé reproductive destinés aux jeunes, dans les régions de l'Afrique subsaharienne sont déplorables et considérés inadéquats (Hughes et McCauley 1998).

Références bibliographiques et publications similaires Alan Guttmacher Institute (AGI). 1998. Into a New World : Young Women's Sexual and Reproductive Lives. New York : AGI.

Bongaarts, John and Barney Cohen. 1998. «Introduction and overview. » Studies in Family Planning 29(2) : 99-105.

Clark, Shelley. 2004. "Early marriage and HIV risks in subSaharan Africa. » Studies in Family Planning 35(3) : 149-160.

Cohen, Susan A. 2004. « Delayed marriage and abstinenceuntil-marriage : On a collision course ? "The Guttmacher Report on Public Policy 7(2) : 1-3.

Hughes, Jane and Ann P. McCauley. 1998. «Improving the fit: Adolescents' needs and future programs for sexual and reproductive health in developing countries. " Studies in Family Planning 29(2) : 233-245.

Mensch, Barbara S. 2005. "The transition to marriage, » in Growing Up Global: The Changing Transitions to Adulthood in Developing Countries, Cynthia B. Lloyd (ed.). Washington, DC : The National Academies Press, pp. 416-505.

Mensch, Barbara S., Monica J. Grant, and Ann K. Blanc. 2006. "The changing context of sexual initiation in subSaharan Africa. " Population and Development Review 32(4) : 699-727. (Earlier version published in 2005 as Policy Research Division Working Paper no. 206. New York : Population Council.)

\section{Donateurs}

Department for International Development (DFID) ; The William and Flora Hewlett Foundation; The Andrew W. Mellon Foundation

\section{Collaborateurs de recherche}

Ann Blanc, MacArthur Foundation ; Shelley Clark, McGill

University ; Monica Grant, University of Pennsylvania 
Pour plus d'informations ou pour des copies d'autres lettres d'information, veuillez contacter publications@popcouncil.org Si vous souhaitez consulter des ressources complémentaires, veuillez consulter www.popcouncil.org/pgy

Population Council

One Dag Hammarskjold Plaza

New York, New York 10017 USA

(c) 2007 The Population Council, Inc. 